\title{
GENERAL RELATIVISTIC ELECTRODYNAMICS OF THE MAGNETIC POLAR REGIONS OF NEUTRON STARS
}

\author{
A. G. Muslimov and A. I. Tsygan \\ A. F. Ioffe Institute of Physics and Technology
}

\begin{abstract}
The induction of the electric fields near a rotating neutron star is considered within the framework of General Relativity. It is demonstrated that within the open magnetic field region, filled by relativistically moving charged particles, a sufficiently strong component of the electric field is generated. This component is due to the effect of dragging of inertial frames of reference and predominates in the case when a neutron star is not exactly an orthogonal rotator. Finally, we discuss some implications of our results on the theory of radio pulsars.
\end{abstract}

\section{Introduction}

Several years ago we studied the electric fields generated near a rotating neutron star in vacuo in General Relativity and discussed some plausible consequences for pulsars (Muslimov and Tsygan 1986). In the present r'-)ort we would like to outline some more recent results (Muslimov and Tsygan 1990a, Muslimov and Tsygan 1990b) on General Relativistic effects which pertain to the electrodynamics of neutron stars. Here we concentrate on a pulsar model like that considered by Scharlemann, Arons and Fawley (1978) and Arons and Scharlemann (1979). The main reason for this is that the alternative models of Sturrock (1971) and Ruderman and Sutherland (1975) confront us with a number of problems which arise if the cohesive energies of atoms in the very strong magnetic fields at the neutron-star surface are much lower (Jones 1986) $(\sim 100 \mathrm{eV})$ than those found previously $(\sim 3 \mathrm{keV})$. Our main purpose here is not to propose a detailed model but to point out the hitherto neglected possibility of General Relativistic effects which generate strong electric fields and electron-positron avalanches above the polar caps of a neutron star.

\section{The basic equations and solutions}

Consider the metric of an asymptotically flat, stationary, axially symmetric spacetime near a rotating neutron star. The non-vanishing components of the metric tensor (in coordinates $x^{0}=c t, x^{1}=R$, $x^{2}=1, x^{3}=\phi$ ) are (Landau and Lifshitz 1975)

$$
g_{00}=-1 / g_{11}=\left(1-r_{\mathrm{g}} / R\right) \equiv \alpha^{2}, g_{22}=-R^{2}, g_{33}=-R^{2} \sin ^{2} \vartheta, g_{03}=w R^{2} \sin ^{2} \vartheta,
$$

where $r_{g}=2 G . M / c^{2}$ is the gravitational radius of a neutron star, $G$ the gravitational constant, $M$ the stellar mass, c the velocity of light, $\omega=2 G J / c^{2} R^{3}$, and $J$ the stellar angular momentum.

In the following we will neglect effects which are quadratic in $J / M c r_{g}$. Bear in mind that the nondiagonal component of the metric, $g_{03}$, leads to the well known effect of dragging of inertial frames of reference with the angular velocity $\omega J / J$ (Bardeen, Press, and Teukolsky 1972).

The equations of electrodynamics will be expressed in terms of absolute (but curved) 3-dimensional space and universal time in accordance with the formalism of Macdonald and Thorne (1982). The electric field, $E$, magnetic field, $B$, electric charge density, $\rho$, and electric current density, $j$, are defined in a ZA.MO (Zero-Angular-Momentum-Observer) frame of reference, which is described by an orthonormal tetrad of 4-vectors.

The Maxwell equations are (Macdonald and Thorne 1982)

$$
\begin{aligned}
\nabla \cdot B & =0 \\
\nabla \times(\alpha E) & =-\frac{1}{c}\left(\frac{\partial B}{\partial t}+\mathcal{L}_{\omega m} B\right), \\
\nabla \cdot E & =4 \pi \rho, \\
\nabla \times(\alpha B) & =\frac{1}{c}\left(\frac{\partial E}{\partial t}+\mathcal{L}_{\omega m} E\right)+\frac{4 \pi}{c} \alpha j,
\end{aligned}
$$

where $\mathcal{L}_{\omega m}$ is the Lie derivative along the vector $w=\omega m$, and $m=r \sin \vartheta e_{\hat{\phi}}$ is the Killing vector of axial symmetry. 
Consider a neutron star rotating with angular velocity $\Omega$ relative to a distant observer and pass to le frame of reference corotating with the star. Then from eq.(2) we obtain

$$
\nabla \times\left(\alpha E-\frac{1}{c}(w-u) \times B\right)=\frac{1}{c} \frac{\partial B}{\partial t}
$$

here $u=\Omega r \sin \vartheta e_{\dot{\phi}}$.

Assuming that the magnetosphere (at least, in the near zone) is stationary in the frame corotating ith a star, on the basis of eq.(5) we can write

$$
\alpha E-\frac{1}{c}(w-u) \times B=-\nabla \Phi,
$$

here the scalar potential, $\Phi$, satisfies the Poisson equation in General Relativity

$$
\boldsymbol{\nabla} \cdot\left(\frac{1}{\alpha} \nabla \Phi\right)=-4 \pi\left(\rho-\rho_{\mathrm{eff}}\right)
$$

here

$$
\rho_{\mathrm{eff}}=\frac{1}{4 \pi c} \nabla \cdot\left(\frac{1}{\alpha}(w-u) \times B\right)
$$

the effective charge density.

For further purposes we will use an adequate approximation, $\vartheta \ll 1$. Then the equation of the last pen magnetic field lines (for the dipole magnetic field in the Schwarzschild background) takes the form Muslimov and Tsyga.n 1989, 1990)

$$
\theta=\theta_{0}[\eta f(1) / f(\eta)]^{1 / 2}
$$

here

$$
\theta_{0}=(\Omega a / c)^{1 / 2} f^{-1 / 2}(1)
$$

the magnetic colatitude of the last open field lines at the stellar surface,

$$
\begin{aligned}
\eta & =R / a, \\
f(\eta) & =-3\left(\frac{\eta}{\varepsilon}\right)^{3}\left[\ln \left(1-\frac{\varepsilon}{\eta}\right)+\frac{\varepsilon}{\eta}\left(1+\frac{\varepsilon}{2 \eta}\right)\right], \\
\varepsilon & =r_{g} / a
\end{aligned}
$$

a compactness parameter, and $a$ is the radial coordinate of the stellar surface.

The explicit expression for the effective charge density is

$$
\rho_{\mathrm{eff}}=-\frac{\Omega B_{0}}{\pi c} \frac{1}{\alpha \eta^{3}}\left(F(\eta) \cos \chi+\frac{3}{2} H(\eta) \vartheta \sin \chi \cos \lambda\right)
$$

there

$$
\begin{aligned}
B_{0} & \equiv B_{\hat{r}}(R=a, \vartheta=0) / 2 f(1) \\
F(\eta) & =f(\eta)\left(1-\kappa / \eta^{3}\right) \\
H(\eta) & =f(\eta)\left(1-(1-\varepsilon / \eta) / \eta-\kappa / \eta^{3}\right)+\frac{1}{\eta-\varepsilon}\left(1-3 \varepsilon / 2 \eta+\kappa / 2 \eta^{3}\right) \\
\kappa & \equiv 2 G J / \Omega a^{3} c^{2}
\end{aligned}
$$

nd where $\kappa$ is the angle between the angular and magnetic momenta of a neutron star, and $\lambda=\phi-\Omega t$.

Here we shall consider space charge limited flow (see, e.g., Scharlemann et al. 1978) with the following tandard boundary conditions for the potential and electric field

i. There exists a closed magnetosphere filled with plasma where $\boldsymbol{E} \cdot \boldsymbol{B}=0$.

ii. The boundary between the closed magnetosphere and the open field lines is a surface of $\Phi=0$. At the stellar surface $\Phi=0$ and $E_{\|}=E \cdot B / B=0$. 
The motions of charged particles $\left(e^{-}, e^{+}\right)$in the intense magnetic field can be approximated as a rela tivistic motion along field lines, with

$$
j=c \rho B / B \text {. }
$$

For the stationary axially symmetric case eqs.(4) and (11) yield

$$
\boldsymbol{B} \cdot \boldsymbol{\nabla}(\alpha \rho / B)=0 \text {. }
$$

Now it is convenient to introduce coordinates $\eta=R / a$ and $\xi=\vartheta / \theta$. Then the solution of eq.(12) is

$$
\rho=-\frac{\Omega B_{0}}{2 \pi c} \frac{1}{\alpha \eta^{3}} f(\eta)\left(k_{1}(1-\kappa) \cos \chi+\frac{3}{2} k_{2} \frac{H(1)}{f(1)} \theta_{0} \xi \sin \chi \cos \lambda\right),
$$

where $k_{1}, k_{2}$ are the constants to be determined.

Consider the solution of eq. (7) near the very surface of a neutron star when $\eta-1=z \ll 1$. In thi case $\nabla_{\|} \cdot E_{\|} \approx(1-\varepsilon)^{1 / 2} a^{-2} \partial^{2} \Phi / \partial z^{2}$. By solving eq.(7). with the above boundary conditions, we ca determine the constants $k_{1}$ and $k_{2}$ from the requirement that at $R-a \geq \delta$ (where $\delta$ is the polar ca radius) this solution is matched with that far from the stellar surface (see below).

The expression for the longitudinal component of the electric field is

$$
\begin{aligned}
& E_{\|}(z, \vartheta) \equiv \boldsymbol{E} \cdot \boldsymbol{B} / B=-\frac{1}{a} \frac{\partial \Phi}{\partial z} \\
&=-\frac{1}{2} B_{0} \theta_{0}^{2}\left(\frac{6 G J}{a^{2} c^{3}} f(1)\left[\sum_{i=1}^{\infty}\left(1-e^{-\beta_{i} z / \theta_{0}(1-\epsilon)^{1 / 2}}\right) \times 8 \frac{J_{0}\left(\beta_{i} \vartheta / \theta_{0}\right.}{\beta_{i}^{3} J_{1}\left(\beta_{i}\right)}\right] \cos \chi\right. \\
&\left.\quad+\frac{\Omega a}{c} \theta_{0} s\left[\sum_{i=1}^{\infty}\left(1-e^{-\gamma_{i} z / \theta_{0}(1-\epsilon)^{1 / 2}}\right) \times 6 \frac{J_{1}\left(\gamma_{i} \vartheta / \theta_{0}\right.}{\gamma_{i}^{3} J_{2}\left(\gamma_{i}\right)}\right] \sin \chi \cos \lambda\right),
\end{aligned}
$$

where

$$
s=H(1) / 2+H^{\prime}(1)=f(1)(1+(3 \varepsilon-\kappa) / 2)-(1+5(\varepsilon-\kappa) / 2-\varepsilon(9 \varepsilon-7 \kappa) / 2) / 2(1-\varepsilon)^{2} .
$$

$\beta_{i}$ and $\gamma_{i}$ are the positive roots of the Bessel functions $J_{0}(x)$ and $J_{1}(x)$, respectively.

We will demonstrate all solutions by setting the constants $k_{1}$ and $k_{2}$ equal to unity. This is justifiec since

$$
k_{1}=1-\frac{3 \theta_{0}(1-\varepsilon)^{1 / 2}}{\beta_{i}(1-1 / \kappa)} \approx 1, \quad k_{2}=1+\frac{\theta_{0}(1-\varepsilon)^{1 / 2}}{\gamma_{i} H(1)} H^{\prime}(1) \approx 1
$$

Now we present the solution above the polar cap when $\theta_{0} \ll \eta-1 \ll c / \Omega a$. In this case we can neglec $\nabla_{\|} \cdot E_{\|}$in Poisson's equation and write $\nabla \cdot E \approx \nabla_{\perp} \cdot E_{\perp}$. Then we obtain the solution

$$
E_{\|}(\eta, \xi)=-\frac{1}{a} \frac{\partial \Phi}{\partial \eta}=-B_{0} \theta_{0}^{2}\left[\frac{6 G J}{a^{2} c^{3}} \frac{f(1)}{\eta^{4}} \cos \chi+\frac{3}{4} \frac{\Omega a}{c} Q(\eta) \theta_{0} \xi \sin \chi \cos \lambda\right]\left(1-\xi^{2}\right)
$$

where

$$
\begin{aligned}
Q(\eta)= & (f(1) / f(\eta))^{3 / 2} \eta^{1 / 2}\left(-f(\eta)\left(1-\left(2-3 \varepsilon / \eta+4 \kappa / \eta^{2}\right) / \eta\right) / \eta+\ldots\right. \\
& +9\left(1-\left(3 \varepsilon-\kappa / \eta^{2}\right) / 2 \eta\right) / 2(\eta-\varepsilon)^{2} f(\eta)+3\left(1-(1-\varepsilon / \eta) / \eta-\kappa / \eta^{3}\right) / 2(\eta-\varepsilon)+\ldots \\
& \left.-\left(1-\left(3 \varepsilon-\kappa / \eta^{2}\right) / 2 \eta\right) /(\eta-\varepsilon)^{2}-\left(8-\left(15 \varepsilon-7 \kappa / \eta^{2}\right) / \eta\right) / 2 \eta(\eta-\varepsilon)\right)
\end{aligned}
$$

with $Q \approx f^{3 / 2}(1) / 2 \eta^{1 / 2}$, when $\eta \gg 1$.

The expression for $E_{\|}$when $z \geq \theta_{0}(1-\varepsilon)^{1 / 2} / \min \left(\beta_{i}, g_{i}\right)$ is

$$
E_{\|}(z, \vartheta) \cong-\frac{1}{2} B_{0} \theta_{0}^{2}\left(\frac{6 G J}{a^{2} c^{3}} f(1) \cos \chi+\frac{3}{8} \frac{\Omega a}{c} \operatorname{si} \sin \chi \cos \lambda\right)\left(1-\vartheta^{2} / \theta_{0}^{2}\right) .
$$

It is easy to see from the above solutions that allowance for the effects of General Relativity leads to new physical result (Muslimov and Tsygan 1989, 1990). Namely, for all inclination angles $\chi \neq \pi / 2$ a electric field proportional to $(6 G J) /\left(a^{2} c^{3}\right)=(6 / 5)[(\Omega a) / c]\left(r_{g} / a\right)\left[(5 I) /\left(2 M a^{2}\right)\right]$ (where $I$ is the momer of inertia of a neutron star) is induced which is $\sim(c / \Omega a)^{1 / 2}$ times greater than that in the case of a $\mathrm{fl}$ spacetime. 


\section{Discussion}

Now we demonstrate that the essentially relativistic electric field under consideration can lead to the formation of an electron-positron avalanche above the polar cap along a path of the order of a stellar radius. In the following estimates we will use the crude approximation $r_{\mathrm{g}} / a \ll 1$.

The value of the accelerating electric field can be estimated as

$$
E_{\|} \simeq 0.5 B_{0}(\Omega a / c)^{2}
$$

for $r_{\mathrm{g}} / a \simeq 0.4$ and $I \simeq(2 / 5) M a^{2}$.

The Lorentz factor of the primary particles is $\gamma=e E_{\|} a / 3 m_{e} c^{2}$, where $e$ is the electric charge of an electron. The critical frequency of the spectrum of curvature radiation is $\omega_{c}=3 c \Gamma^{3} / 2 R_{c u r v}$, where $R_{\text {curv }} \cong(4 / 3) a(c / \Omega a)^{1 / 2}$ is the radius of curvature of a polar field line.

Now writing the condition for pair creation

$$
\frac{\hbar \omega_{\mathrm{c}}}{2 m_{\mathrm{e}} c^{2}} \frac{B}{B_{\mathrm{c}}} \frac{a}{R_{\text {curv }}} \sim 0.1
$$

when $\left(\hbar \omega_{\mathrm{c}}\right) /\left(m_{\mathrm{e}} c^{2}\right)\left(a / R_{\text {curv }}\right) \gg 1$. Here $B_{\mathrm{c}} \cong 4.4 \times 10^{13} \mathrm{G}$ is the quantum magnetic field.

The above condition is satisfied for $B=10^{12} \mathrm{G}$ and pulsar rotation period $P=0.5 \mathrm{~s}$, when photons with the critical energy of the curvature spectrum, $\hbar \omega_{c}$, are absorbed. The number of such photons, emitted by primary particles moving along a path $a$, is of the order of $3 \times 10^{2}$. The period of pulsar rotation for which pair formation ceases may be estimated as (for $r_{\mathrm{g}} / a \ll 1$ )

$$
P=P_{\mathrm{c}} B_{12}^{4 / 7}
$$

where $P_{\mathrm{c}} \sim 0.5 \mathrm{~s}$ and $B_{12}=B / 10^{12} \mathrm{G}$.

The total power going into particle acceleration can now be estimated as

$$
\mathcal{P} \approx 0.4 \frac{r_{g}}{a} B^{2} a^{\dot{2}} c\left(\frac{\Omega a}{c}\right)^{4}\left(\frac{5 I}{2 M a^{2}}\right)
$$

which is of the order of that lost by a rotating neutron star from magnetic dipole radiation. 